University of Nebraska - Lincoln

DigitalCommons@University of Nebraska - Lincoln

Virology Papers

Virology, Nebraska Center for

2010

Chlorella viruses encode most, if not all, of the machinery to glycosylate their glycoproteins independent of the endoplasmic reticulum and Golgi

James L. Van Etten

James Gurnon

Giane M. Yanai-Balser

David Dunigan

Michael V. Graves

Follow this and additional works at: https://digitalcommons.unl.edu/virologypub

Part of the Biological Phenomena, Cell Phenomena, and Immunity Commons, Cell and Developmental Biology Commons, Genetics and Genomics Commons, Infectious Disease Commons, Medical Immunology Commons, Medical Pathology Commons, and the Virology Commons

This Article is brought to you for free and open access by the Virology, Nebraska Center for at DigitalCommons@University of Nebraska - Lincoln. It has been accepted for inclusion in Virology Papers by an authorized administrator of DigitalCommons@University of Nebraska - Lincoln. 
Review

\title{
Chlorella viruses encode most, if not all, of the machinery to glycosylate their glycoproteins independent of the endoplasmic reticulum and Golgi
}

\author{
James L. Van Etten, ${ }^{1}$ James R. Gurnon, ${ }^{1}$ \\ Giane M. Yanai-Balser, ${ }^{1}$ David D. Dunigan, ${ }^{1}$ \\ and Michael V. Graves ${ }^{2}$ \\ 1 Department of Plant Pathology and Nebraska Center for Virology, \\ University of Nebraska, Lincoln, NE 68583-0900, USA \\ 2 Department of Biological Sciences, University of Massachusetts-Lowell, \\ Lowell, MA 01854, USA \\ Corresponding author — J. L. Van Etten, email jvanetten1@unl.edu
}

\begin{abstract}
In contrast to all other viruses that use the host machinery located in the endoplasmic reticulum and Golgi to glycosylate their glycoproteins, the large dsDNA-containing chlorella viruses encode most, if not all, of the components to glycosylate their major capsid proteins. Furthermore, all experimental results indicate that glycosylation occurs independent of the endoplasmic reticulum and Golgi.
\end{abstract}

Keywords: Chlorella viruses, PBCV-1, virus-encoded glycosyltransferases, cytoplasmic glycosylation, virus major capsid protein

Published in Biochimica et Biophysica Acta 1800 (2010), pp 152-159.

doi:10.1016/j.bbagen.2009.07.024

Copyright (C) 2009 Elsevier B.V. Used by permission.

Submitted 22 April 2009; revised 15 July 2009; accepted 18 July 2009; published 3 August 2009. 


\section{Virus-encoded glycosyltransferases}

Structural proteins of many viruses, such as rhabdoviruses, herpesviruses, poxviruses, and paramyxoviruses are glycosylated. Most virus glycoproteins, which can have very complex glycans, are linked to Asn via Nacetylglucosamine, although some viruses have 0-linked sugars attached to Ser or Thr residues via an amino sugar, usually acetylglucosamine or acetylgalactosamine. Typically, viruses use host-encoded glycosyltransferases and glycosidases located in the endoplasmic reticulum (ER) and Golgi apparatus to add and remove N-linked sugar residues from virus glycoproteins either co-translationally or shortly after translation of the protein. This post-translational processing aids in protein folding and involves other host-encoded enzymes such as protein disulfide isomerase. After folding and assembly, virus glycoproteins are transported by host sorting and membrane transport functions to virus specified regions in host membranes where they displace host glycoproteins. Progeny viruses then bud through these virus-specific target membranes, which is usually the final step in the assembly of infectious virions [1-4]. Thus, nascent viruses only become infectious by budding through the membrane, usually the plasma membrane, as they are released from the cell. Consequently, the glycan portion of virus glycoproteins is host-specific. The theme that emerges from these studies is that virus glycoproteins are synthesized and glycosylated by the same mechanisms as host glycoproteins. Therefore, the only way to alter glycosylation of virus proteins is to either grow the virus in a different host or have a mutation in the virus protein that alters the protein glycosylation site.

One outcome of this scenario is that, in general, viruses lack genes encoding glycosyltransferases. However, a few virus-encoded glycosyltransferases have been reported in the last few years (see review [5]). Sometimes these virus-encoded glycosyltransferases add sugars to compounds other than proteins. For instance, some phage-encoded glycosyltransferases modify virus DNA to protect it from host restriction endonucleases (e.g., [6-8]), and a glycosyltransferase encoded by baculoviruses modifies a host insect ecdysteroid hormone leading to its inactivation [9]. Bovine herpesvirus- 4 encodes a $\beta$-1,6-N-acetyl-glucosaminyltransferase that is localized in the Golgi and is probably involved in post-translational modification of virus structural proteins [10-12]. Additional putative virus-encoded glycosyltransferases of unknown function include 
certain pox viruses [13,14], Ectocarpus siliculosus virus 1 [15], Ostreococus virus OsV5 [16], Acanthamoeba polyphaga Mimivirus [17], and two Archaea viruses, Acidianus filamentous virus 3 [18], and Sulfolobus virus STSV1 [19]. With the rapid increase in sequencing virus genomes, additional virus-encoded glycosyltransferases will undoubtedly be discovered.

One group of viruses that differs from the scenario that viruses use the host machinery located in the ER and Golgi to glycosylate their glycoproteins is the chloroviruses (family Phycodnaviridae) that infect eukaryotic algae. Phycodnaviruses are large (150 to $190 \mathrm{~nm}$ in diameter) polyhedral, dsDNA-containing viruses with an internal lipid membrane (genomes of 170 to $560 \mathrm{~kb}$ ) [20-23]. They are present in aqueous environments throughout the world and play dynamic, albeit largely unknown, roles in regulating algal communities in aqueous environments, such as the termination of massive algal blooms commonly referred to as red and brown tides (e.g., [24-27]). Some phycodnaviruses encode as many as 600 proteins, which is more protein-encoding genes than in the smallest bacteria.

The phycodnaviruses probably have a common ancestor with the poxviruses, iridoviruses, African swine fever virus, and Mimivirus (e.g., [28-30]), and accumulating evidence indicates that the phycodnaviruses have a long evolutionary history, possibly dating from the time eukaryotes arose from prokaryotes (2-3billion years ago) (e.g., [30-32]). Some algal virus genes encode commercially important enzymes such as DNA restriction endonucleases (e.g., [33,34]), whereas other viral genes encode proteins that are the smallest in their class and may represent the minimal catalytic unit. Consequently, these 'small' proteins serve as models for mechanistic and structural studies (e.g., DNA topoisomerase type II [35] and a $\mathrm{K}^{+}$ion channel protein [36]).

Phycodnavirus members in the genus Chlorovirus are plaque forming viruses that infect certain isolates of unicellular, chlorella-like green algae. The prototype chlorella virus Paramecium bursaria chlorella virus (PBCV-1) infects a host, Chlorella NC64A, that is normally a symbiont in the protozoan P. bursaria. The $330 \mathrm{~kb}$ PBCV-1 genome has $\sim 690$ potential open reading frames (ORFs) of 65 codons or larger [20]. About 365 of these ORFs are predicted to encode proteins (CDSs) and the functions of $35-40 \%$ of these CDSs have been tentatively identified. Pertinent to this review is that 17 of the PBCV-1 genes encode enzymes that 
manipulate sugars, many of which have never been found previously in a virus (Table 1). At least five of the enzymes are putative glycosyltransferases, presumably involved in glycosylation of the PBCV-1 major capsid protein Vp54. [Note: in our original report on the annotation of the PBCV-1 genome we identified 7 PBCV-1 encoded glycosyltransferases [37]. However, two of these CDSs, which were co-linear with an adjacent glycosyltransferase, were the result of sequencing errors and are part of the adjacent glycosyltransferase.]

Table 1. Virus PBCV-1 encoded enzymes involved in sugar manipulation.

\begin{tabular}{|c|c|c|c|c|}
\hline Activity & $C D S$ & $\begin{array}{l}\text { Size } \\
(a a)\end{array}$ & $\begin{array}{c}\text { Expressed }^{a} \\
\text { (min p.i.) }\end{array}$ & Reference \\
\hline \multicolumn{5}{|l|}{ Glycosyltransferases } \\
\hline & A064R & 638 & $20-60$ & [60] \\
\hline & $\mathrm{A} 111 / 114 \mathrm{R}$ & 860 & $40-60$ & - \\
\hline & A219/222/226R & 677 & $20-60$ & - \\
\hline & A473L & 517 & $20-60$ & - \\
\hline & A546L & 396 & $40-60$ & - \\
\hline \multicolumn{5}{|l|}{ Hyaluronan biosynthesis } \\
\hline UDP-glucose dehydrogenase & A609L & 389 & $20-40$ & [42] \\
\hline Glutamine:fructose-6- $\mathrm{PO}_{4}$ amidotransferase & A100R & 595 & $20-60$ & [42] \\
\hline Hyaluronan synthase & A098R & 568 & $20-40$ & [38] \\
\hline \multicolumn{5}{|l|}{ Sugar nucleotide biosynthesis } \\
\hline GDP-D-mannose 4,6 dehydratase & A118R & 345 & $40-90$ & [46] \\
\hline Fucose synthase $\mathrm{e}^{\mathrm{b}}$ & A295L & 317 & $40-90$ & [46] \\
\hline \multicolumn{5}{|l|}{ Polysaccharide degrading enzymes } \\
\hline Chitinase & A181/182R & 830 & $60-360$ & [49] \\
\hline Chitinase & A260R & 484 & $90-360$ & [49] \\
\hline Chitosanase & A292L & 328 & $90-360$ & [49] \\
\hline$\beta$-1,3-glucanase & A094L & 364 & $20-40$ & [50] \\
\hline$\beta$ and $\alpha 1,4$-linked glucuronic lyase & A215L & 321 & $120-360$ & {$[51]$} \\
\hline \multicolumn{5}{|l|}{ others } \\
\hline D-lactate dehydrogenase & A053R & 363 & $20-60$ & - \\
\hline Pyrimidine dimer-specific glycosylase & A050L & 141 & $20-60$ & [52] \\
\hline
\end{tabular}

a. The time the genes were expressed was determined by microarray analysis (Yanai-Balser, G.M. et al., manuscript in preparation).

b. Another name is GDP-4-keto-6-deoxy-D-mannose epimerase reductase. 


\section{Chlorovirus-encoded sugar enzymes}

Three PBCV-1 encoded enzymes are involved in the synthesis of the extracellular matrix polysaccharide hyaluronan (also referred to as hyaluronic acid), including the glycosyltransferase hyaluronan synthase (HAS) [38,39]. Hyaluronan, a ubiquitous constituent of the extracellular matrix in vertebrates, consists of $\sim 20,000$ alternating $\beta$-1,4-glucuronic acid and $\beta-1,3-\mathrm{N}$-acetylglucosamine residues [40]. Hyaluronan synthesis occurs at the plasma membrane and is simultaneously extruded through the membrane to the exterior of the cell. Until the has gene was discovered in PBCV-1, hyaluronan was thought to only occur in vertebrates and a few pathogenic bacteria, where it forms an extracellular capsule, presumably to avoid the immune system $[40,41]$.

PBCV-1 also encodes two enzymes involved in the biosynthesis of hyaluronan precursors, glutamine:fructose-6-phosphate amidotransferase and UDP-glucose dehydrogenase [42]. All three genes are expressed early during PBCV-1 infection. These results led to the discovery that hyaluronan lyase-sensitive hair-like fibers begin to accumulate on the surface of PBCV-1 infected host cells by 15 min post infection (p.i.). By 4 h p.i., the infected cells are covered with a dense fibrous hyaluronan network [39].

The has gene is present in many, but not all, chloroviruses isolated from diverse geographical regions [39]. Surprisingly, many chloroviruses that lack a has gene have a gene encoding a functional chitin synthase (CHS). Furthermore, cells infected with these viruses produce chitin fibers on their external surface [43]. Chitin, an insoluble linear homopolymer of $\beta$-1,4-linked $\mathrm{N}$-acetyl-glucosamine residues, is a common component of insect exoskeletons, shells of crustaceans and fungal cell walls [44].

A few chloroviruses contain both has and chs genes, and form both hyaluronan and chitin on the surface of their infected cells $[43,45]$. Finally, a few chloroviruses probably lack both genes because no extracellular polysaccharides are formed on the surface of cells infected with these viruses [39]. The fact that many chloroviruses encode enzymes involved in extracellular polysaccharide biosynthesis suggests that the polysaccharides, which require a huge expenditure of ATP for their synthesis, are important in the virus life cycle. However, at present this function(s) is unknown. 
The chloroviruses encode additional enzymes involved in nucleotide sugar metabolism. Two enzymes encoded by virus PBCV-1, GDP-Dmannose 4,6 dehydratase (GMD) and a GDP-4-keto-6-deoxy-D-mannose epimerase reductase (GMER) comprise the highly conserved pathway that converts GDP-D-mannose to GDP-L-fucose. In vitro reconstruction of the biosynthetic pathway using recombinant PBCV-1 GMD and GMER synthesized GDP-L-fucose [46,47]. Unexpectedly, however, the PBCV-1 GMD also catalyzes the NADPH-dependent reduction of the intermediateGDP-4-keto-6-deoxy-D-mannose, forming GDP-D-rhamnose. Both fucose and rhamnose are constituents of the glycans attached to the PBCV-1 major capsid protein Vp54 (see below). Therefore, the virus might encode the enzymes to meet this need. Other chloroviruses encode additional sugar-metabolizing enzymes including a putative mannose-6-phosphate isomerase and a UDP-glucose 4,6 dehydratase [48] (Tonetti, M. et al., unpublished data).

At least five PBCV-1-encoded enzymes are involved in polysaccharide degradation, including 2 chitinases, a chitosanase, a $\beta$-1,3-glucanase, and a $\beta$ - and $\alpha$-1,4-linked glucuronic acid lyase (summarized in [21], [4951]). Finally, one enzyme is a glycosylase that initiates pyrimidine photodimer excision $[52,53]$.

\section{Glycosylation of virus PBCV-1 major capsid protein Vp54}

The major capsid protein of PBCV-1, Vp54 (comprises 40\% of total virus protein), is a glycoprotein with a molecular weight of 53,790 (Table 2). Glycosylation of Vp54 and two minor structural proteins Vp280 ( 1\% of the total virus protein) and Vp260 ( $\sim 0.1 \%$ of total virus protein) differs from other viruses. CDS A430L encodes Vp54 with a predicted molecular weight of 48,165 Da. This protein undergoes additional post-translational modifications in addition to being glycosylated. The aminoterminal Met is removed [54] and the protein is myristylated in the carboxyl-terminal portion of the protein, probably at an internal Lys or Arg [55]. Vp260 is coded by CDS A122/123R, a protein of $138 \mathrm{kDa}$. Since the protein migrates on SDS-PAGE with a weight of $\sim 260 \mathrm{kDa}$, it probably consists of more than $40 \%$ carbohydrate. The CDS encoding Vp280 is unknown. 
Table 2. Properties of antigenic classes of viruses derived from PBCV-1. Modified from [60].

\begin{tabular}{|c|c|c|c|c|c|}
\hline \multicolumn{3}{|c|}{ Antigenic classes } & \multicolumn{3}{|c|}{ Properties of members of each antigenic class } \\
\hline Class & $\begin{array}{l}\text { Proposed } \\
\text { genotype }\end{array}$ & $\begin{array}{r}M r V p 54 \\
S D S-P A G E\end{array}$ & Virus & $\begin{array}{r}\text { MW Vp54, mass } \\
\text { spectrometry }\end{array}$ & $\begin{array}{r}\text { Total glycan } \\
\text { mass }^{a}\end{array}$ \\
\hline Wild-type & wt & $54 \mathrm{kDa}$ & PBCV-1 & 53,790 & 5545 \\
\hline P91 & $m p 91$ & $53 \mathrm{kDa}$ & P91 & 52,780 & 4535 \\
\hline \multirow[t]{6}{*}{ EPA-1 } & тера1 & $52 \mathrm{kDa}$ & EPA-1 & 51,560 & 3315 \\
\hline & & & EPA-2 & $n d^{b}$ & nd \\
\hline & & & EPA-3 & nd & nd \\
\hline & & & P31 & 51,593 & 3348 \\
\hline & & & P1050 & nd & nd \\
\hline & & & P1056 & nd & nd \\
\hline \multirow[t]{4}{*}{ E11 } & mel 1 & $51 \mathrm{kDa}$ & E11 & nd & nd \\
\hline & & & $\mathrm{P} 41$ & nd & nd \\
\hline & & & P1210 & nd & nd \\
\hline & & & P1219 & nd & nd \\
\hline P100 & mp100 & $50.5 \mathrm{kDa}$ & P100 & 51,038 & 2793 \\
\hline
\end{tabular}

a. Calculated by subtracting the deduced peptide mass minus the N-terminal initiating Met residue $(48,017)$ and the mass of one myristate $(228.4)$ from the MW determined by mass spectrometry. All the protein backbones are identical.

b. nd, not determined.

Cryo-electron microscopy and three-dimensional (3-D) icosahedral image reconstruction of the PBCV-1 virion indicate that the outer capsid is icosahedral and covers a lipid bilayered membrane [56,57]. The capsid shell consists of 1680 donut-shaped trimeric capsomers plus 12 pentameric capsomers at each icosahedral vertex. The trimeric capsomers are arranged into 20 triangular facets (trisymmerons, each containing 66 trimers) and 12 pentagonal facets (pentasymmetrons, each containing 30 trimers and one pentamer at the icosahedral vertices). Trisymmetrons and pentasymmetrons are made up of trimeric capsomers, with each doughnut shaped capsomer composed of 3 monomers of Vp54. The trimeric capsomers are easy to isolate because heating PBCV-1 to $65^{\circ} \mathrm{C}$ for a few minutes leads to solubilization of the trimers. However, the monomer form of Vp54 is insoluble.

Recent five-fold symmetry, rather than icosahedral, averaging of cryoelectron microscopy data has produced some exciting results on the structure of PBCV-1 [58]. PBCV-1 has a unique pentameric vertex with 
a $250 \AA$ Å long and $50 \AA$ wide cylindrical spike that connects to a large pocket between the capsid and the enveloped nucleocapsid. Circumstantial evidence suggests that Vp260 may be a component of the spike structure, which presumably is involved in virus attachment.

The conclusion that the PBCV-1 proteins are glycosylated by a different mechanism than those used by other viruses originally arose from antibody studies. Polyclonal antiserum prepared against intact PBCV-1 virions inhibits virus plaque formation by agglutinating particles. Spontaneously derived, antiserum-resistant, plaque-forming variants of PBCV-1 occur at a frequency of $10^{-5}$ to $10^{-6}$. These antiserum-resistant variants fall into four serologically distinct classes (Table 2) [59]. Polyclonal antisera prepared against members of each of these antigenic classes react exclusively or predominately with the Vp54 (and Vp280 and Vp260) equivalents from the viruses in the class used for the immunization. While each of the three glycoproteins from the antigenic variants migrate faster on SDS-PAGE than those of the strains from which they are derived, all of the major capsid proteins co-migrate after deglycosylation. Western blot analyses of Vp54 proteins isolated from the variants, before and after removing the glycans with trifluoromethane-sulfonic acid or altering the glycan with periodic acid, established that the antigenic variants reflect differences in the Vp54 glycans. In addition, the ratio of the seven neutral sugars [glucose, fucose, galactose, mannose, xylose, rhamnose and arabinose [59] attached to PBCV-1 Vp54 and the variants change in a manner that correlates with antigenicity and Vp54 migration on SDS-PAGE (Table 3). Variants from different classes can complement and recombine in dual infection experiments to produce wild-type progeny, indicating that the enzymes involved in glycosylation reside in different virus-encoded complementation groups [60].

Additional observations indicate that Vp54 glycosylation is unusual: i) unlike viruses that acquire their glycoprotein(s) by budding through a plasma membrane, intact infectious PBCV-1 particles accumulate inside the host 30-40 min before virus release [61]. ii) The nucleotide sequence of the Vp54 gene ( $a 430 l$ ) in each of the variants is identical to wild-type PBCV-1, thus the peptide portion of Vp54 is not altered in the mutants [59]. iii) All of the antigenic variants are grown in the same host so the differences cannot be attributed to the host. iv) Polyclonal antibodies to Vp54 do not react with host glycoproteins. v) Neither N-acetylglucosamine (GlcNAc) nor $\mathrm{N}$-acetylgalactosamine was detected in Vp54 
glycans, sugars commonly found in Asn-linked (N-linked) and many Ser/ Thr-linked (0-linked) glycoproteins produced via the cellular ER-Golgi pathway (Table 3) [59]. vi) Unlike most glycoproteins that exhibit size microheterogeneity, Vp54 appears homogeneous on SDSPAGE; in addition, mass spectrometry analysis only reveals one satellite peak that differs in molecular weight by $140 \mathrm{Da}$, the approximate weight of either one arabinose, xylose, fucose or rhamnose sugar (Cerny, R. and Van Etten, J.L., unpublished data). vii) The ability to easily crystallize Vp54 as a homotrimer provides additional evidence that the protein is essentially homogeneous (Fig. 1) [62].

The glycans are not linked to the major capsid proteins by a traditional N-linkage because: i) treatment of Vp54 with the enzymes endo- $\beta$ $\mathrm{N}$-acetylglucosaminidase $\mathrm{F}$ or $\mathrm{N}$-glucosidase $\mathrm{F}$, which cleave glycans from $\mathrm{N}$-linked glycoproteins [63], does not alter Vp54 migration on SDS-PAGE (Chaney, W.G. and Van Etten, J.L., unpublished data). ii) The drug tunicamycin, which inhibits synthesis of N-linked glycoproteins [64], has no effect on PBCV-1 replication nor Vp54 electrophoretic mobility, even at concentrations three times that required to inhibit host chlorella growth [55]. Finally, an antibody (provided by Felix Wieland) to a $\beta$-glucose Asn linkage present in bacterial glycoproteins [65] reacts strongly with Vp54 (Gurnon, J.R. and Van Etten, J.L., unpublished data), suggesting that one

Table 3. Sugar composition of the glycoproteins from PBCV-1 serotypes. (Reproduced from [59]).

\begin{tabular}{lccccccc} 
Sugar & \multicolumn{7}{l}{ Viruses - relative sugar content } \\
\cline { 2 - 8 } & $P B C V-1$ & $P 91$ & $E P A-1$ & $P 31$ & $P 41$ & $E 11$ & $P 100$ \\
\hline Fucose & 0.69 & 0.73 & 0.88 & 0.81 & 0.78 & 0.83 & 0.61 \\
Ara/Rham $^{\mathrm{a}}$ & 1.57 & 1.24 & 0.64 & 0.65 & 0.65 & 0.65 & 0.19 \\
Galactose $_{\text {Glucose }}$ & 1.02 & 0.97 & 1.02 & 1.03 & 0.99 & 0.97 & 0.91 \\
Xylose & 1.00 & 1.00 & 1.00 & 1.00 & 1.00 & 1.00 & 1.00 \\
Mannose & 1.58 & 1.47 & 1.59 & 1.57 & 0.88 & 0.77 & 0.60 \\
& 1.02 & 0.70 & 0.14 & 0.17 & 0.24 & 0.15 & 0.12 \\
\hline
\end{tabular}

Values are normalized to that of glucose. The mutants are arranged so that the major capsid protein decreases in size from PBCV-1 to P100. Viruses EPA-1 and P31 and viruses P41 and E11 are serologically similar even though they are separate isolates. Viruses P91, EPA-1, P31 and P41 are derived from PBCV-1; virus E11 is derived from EPA-1; and virus P100 is from P31. Ara/Rham, arabinose/rhamnose.

a. Subsequent experiments established that both Ara and Rham are present in the PBCV-1 major capsid protein. 


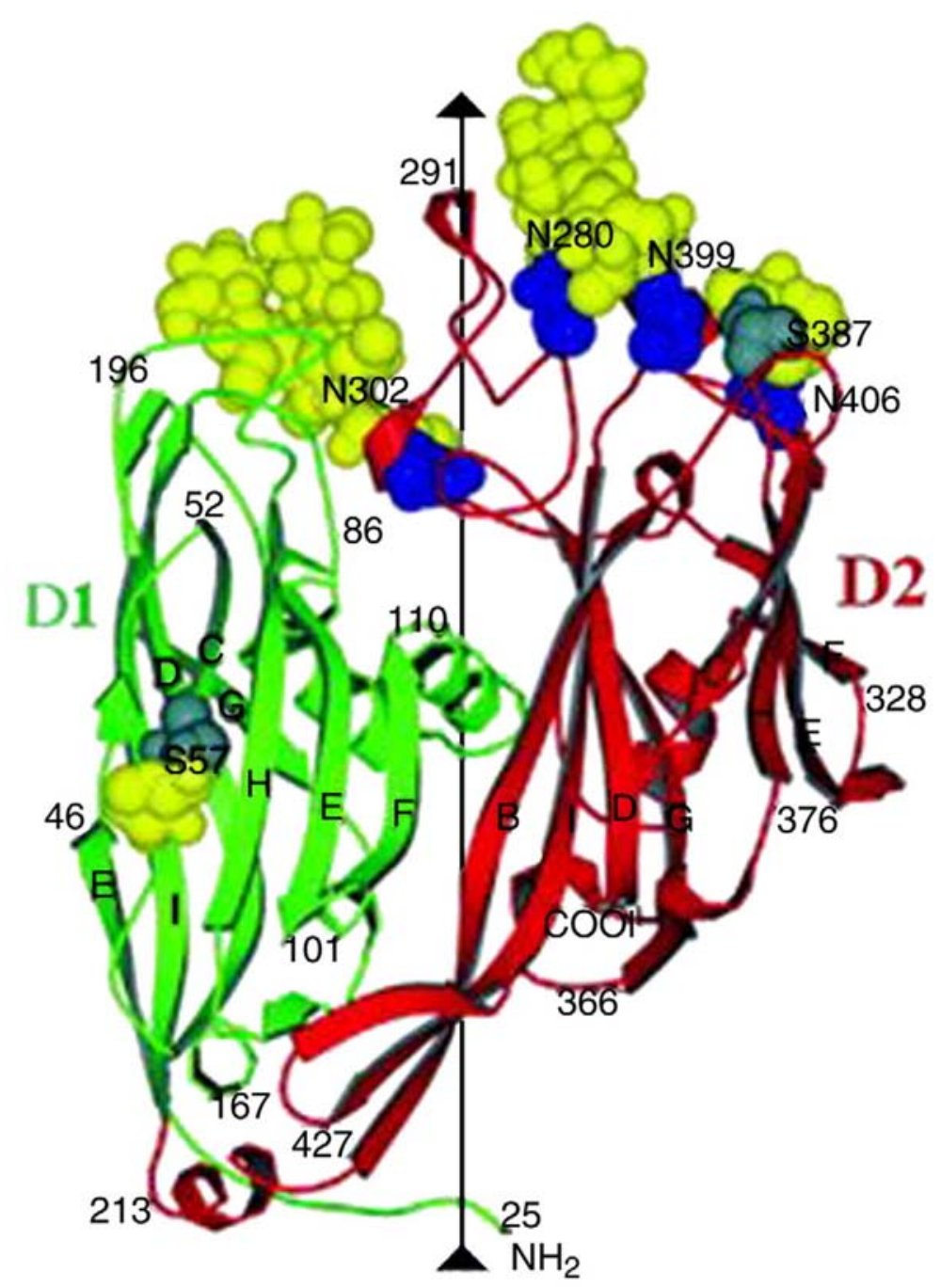

Fig. 1. A ribbon diagram of the crystal structure of the virus PBCV-1 major capsid protein, Vp54, monomer with strategic amino acids labeled. The carbohydrate moieties (yellow) and glycosylated Asn and Ser residues (blue and gray, respectively) are shown as space-filling atoms. The green and red regions indicate the consecutive "jelly roll" domains of the protein. The arrow shows the orientation of the protein from the inside to the outside of the virus. Reproduced from [62].

or more of the Asn-linked glycans in Vp54 have this linkage. This same linkage exists in the B2 chain of mammalian laminin, a major basement membrane glycoprotein [66].

We originally suggested that Vp54 had a single $\sim 30$ mer glycan or two-15mer glycans attached to it [60]. However, the Vp54 crystal structure revealed sugars attached to six amino acids in the protein (Fig. 1) [62]. Comparison of the molecular mass of Vp54 (53,790 Da) with its 
predicted molecular weight from the amino acid sequence $(48,165 \mathrm{Da})$ indicates that the protein contains 30-35 sugar moieties, of which 20 could be detected, but not identified, in the crystal density map. At a minimum, single sugars were found attached to Ser ${ }^{57}$ and Ser ${ }^{387}$; six and seven-branched chain sugar moieties were found attached to Asn ${ }^{280}$ and $\mathrm{Asn}^{302}$, respectively, as well as three and two sugar residues were located at $\mathrm{Asn}^{399}$ and $\mathrm{Asn}^{406}$, respectively. As predicted from the antibody results, the four $\mathrm{N}$-linked glycans are on the external surface of the virus. GlcNAc fits the carbohydrate electron density at residues $\mathrm{Asn}^{280}$, $\mathrm{Asn}^{302}$, and $\mathrm{Asn}^{399}$. However, the inability to detect GlcNAc in Vp54 suggests that another modified sugar might be in this position, e.g., glucose with an 0-linked acetate.

The identification of the glycan-linked Asn residues in Vp54 provided additional evidence that Vp54 glycosylation does not involve host glycosyltransferases. $\mathrm{Asn}^{302}, \mathrm{Asn}^{399}$, andAsn ${ }^{406}$ occur in the amino acid sequence (A/G)NTXT, and Asn ${ }^{280}$ occurs in an ANIPG sequence. None of these Asn residues resides in a NX(T/S) sequence commonly recognized by ER located glycosyltransferases [67]. These findings also explain why previous enzymatic tests for N-glycosylation were negative. Finally, the major capsid protein, like the glycosyltransferases, lacks an ER or Golgi signal peptide. Therefore, taken together, the results suggest that PBCV-1 encodes some, if not all, of the enzymes involved in constructing the glycans attached to Vp54.

\section{PBCV-1 encoded glycosyltransferases}

As mentioned above, five putative glycosyltransferase-encoding genes have been found in PBCV-1, which are scattered throughout the PBCV-1 genome (Table 1). None of these PBCV-1 encoded glycosyltransferases has an identifiable signal peptide that would target them to the ER. Furthermore, the cellular protein localization program PSORT predicts that all of these proteins, with the exception of A473L, are located in the cytoplasm. A473L is predicted to contain one transmembrane domain.

The genes for all five PBCV-1 encoded glycosyltransferases are expressed early during PBCV-1 infection (Yanai-Balser, G.M. et al., manuscript in preparation). Thus, assuming the proteins are stable, the enzymes should be available for either adding sugars to the Vp54 glycans or transferring the glycans. The $a 4301$ gene is expressed late. 


\subsection{PBCV-1 a64r gene}

The PBCV-1 a64r gene encodes a 638 amino acid protein with 4 motifs that are conserved in the "fringe-class" of glycosyltransferases $[68,69]$. Five conserved domains, designated 1-5, have been identified in this class of enzymes [69]. Domains 3 and 5 contain the proposed catalytic amino acids, the "DXD" sequence in domain 3 and the first " $D$ " residue in domain 5. The A64R protein contains domains 2-5 although the spacing between some of the domains differs from that of fringe-glycosyltransferases. As mentioned above, the A64R protein lacks both an identifiable signal peptide that would target the protein to the ER and a membranespanning domain in contrast to fringe-glycosyltransferases.

Analysis of 13 PBCV-1 antigenic variants revealed mutations in $a 64 r$ that correlated with a specific antigenic class, EPA-1 (Table 2). The $a 64 r$ gene in six of these antigenic variants was sequenced to determine if mutations in a64r correlated with the EPA-1 antigenic variation [60]. The a64r sequences from mutants EPA-1, EPA-3, and P31 have single nucleotide substitutions, which produce a single amino acid substitution in the A64R protein. Two of the amino acid substitutions occur in the DXD motif (domain 3) and the other one is in domain 4. A fourth variant has an extra base in the coding sequence, which creates a frame shift mutation in the gene. Finally, the entire gene is deleted in the other two antigenic variants.

Dual infection experiments with the different antigenic variants indicate that viruses containing wild-type a64r complement and recombine with viruses that contain variant $a 64 r$ to form wild-type virus. Therefore, we concluded that $a 64 r$ encodes a glycosyltransferase involved in the synthesis of the Vp54 glycan [60].

\section{Structure of PBCV-1 encoded A64R glycosyltransferase}

Blast searches with the 638 amino acid A64R protein established that the glycosyltransferase domains are located in the amino-terminal 211 amino acids. The A64R carboxyl terminus resembles several bacterial proteins of unknown function.

The amino-terminal A64R glycosyltransferase domain (residues 1-211) was expressed in a bacterial system for crystallization studies. The crystal structure of the peptide was solved at $1.6 \AA$ resolution 


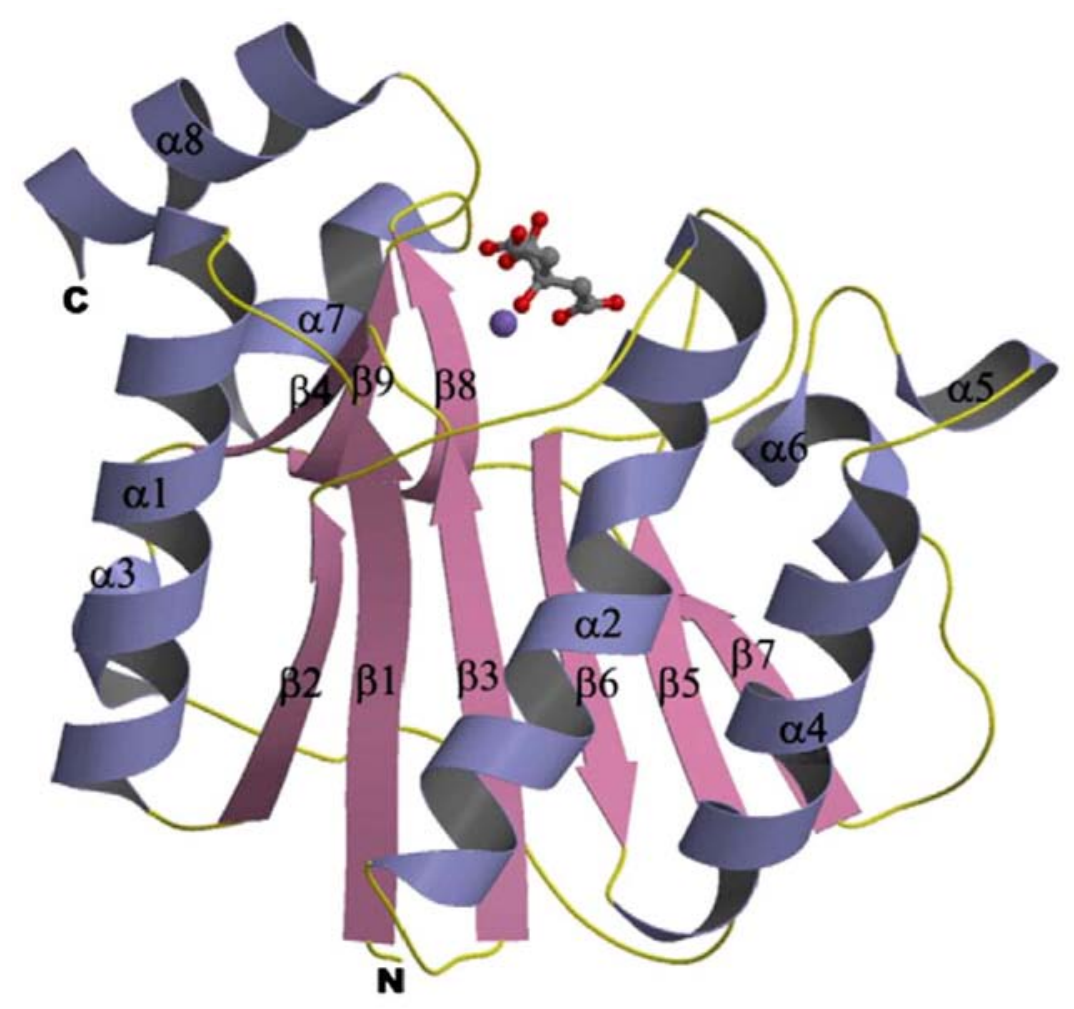

Fig. 2. A ribbon diagram of the crystal structure of the glycosyltransferase domain from virus PBCV-1 CDS A64R. Bound $\mathrm{Mn}^{2+}$ and citrate ions are shown in a ball-and-stick representation. Alpha (purple) and beta sheets (pink) are color coded.

(Fig. 2) [70]. The peptide has a mixed $\alpha / \beta$ fold containing a central, sixstranded $\beta$ sheet flanked by $\alpha$ helices. The overall fold is similar to catalytic domains of glycosyltransferases in the GT-A group, retaining glycosyltransferase family 34 , although the amino acid similarity between them is low (less than $14 \%$ for equivalent $C \alpha$ atoms). Crystal structures of A64R complexed with UDP, CMP, or GDP, established that only UDP bound to $\mathrm{A} 64 \mathrm{R}$ in the presence of $\mathrm{Mn}^{2+}$, consistent with its high structural similarity to glycosyltransferases that use UDP as the sugar carrier. The structure of the A64R, UDP-glucose, and $\mathrm{Mn}^{2+}$ complex showed that the largest conformational change occurred when hydrogen bonds were formed with the ligands. Unlike UDP-glucose, UDP-galactose and UDP-GlcNAc did not bind to A64R, suggesting a selective binding of UDPglucose. Thus UDP-glucose is most likely the sugar donor for A64R, consistent with glucose occurring in the virus major capsid protein glycans (Table 3). 
However, no enzymology has been conducted on the A64R peptide, or any of the other virus-encoded glycosyltransferases because the structure of the Vp54 glycans is unknown. Consequently, the substrate(s) for A64R or any of the putative viral glycosyltransferases are unknown.

\section{Where does Vp54 acquire its glycans?}

A brief description of the PBCV-1 life cycle follows. PBCV-1 infects its host Chlorella NC64A by attaching rapidly, probably at a unique virus vertex $[58,71]$, to the algal cell wall, followed by degradation of the wall at the point of attachment [72]. After digestion of the wall, the PBCV-1 internal membrane presumably fuses with the host membrane to translocate virus DNA and probably some virus associated proteins to the inside of the host, leaving an empty capsid on the surface.

Two observations suggest that upon infection PBCV-1 DNA and probably DNA-associated proteins quickly move to the nucleus and commandeer at least some of the host transcription machinery to initiate early viral RNA synthesis; early virus transcripts can be detected within 5-10 min p.i. [73,74]. i) PBCV-1 does not encode a recognizable RNA polymerase gene(s) nor is RNA polymerase activity detected in isolated virions (Rohozinski, J. and Van Etten, J.L., unpublished data). ii) A small intron with splice-site sequences characteristic of nuclear-spliced mRNAs exists in the PBCV-1 DNA polymerase gene [75]. Presumably, this intron is excised in the cell nucleus.

Viral DNA synthesis begins between 60 and 90 min p.i., followed by late viral transcription $[73,76]$. Virus assembly begins $\sim 3 \mathrm{~h}$ p.i. in localized regions of the cytoplasm called virus assembly centers. By 6$8 \mathrm{~h}$ p.i., virus induced host cell lysis occurs and results in the release of progeny viruses $(\sim 1000$ virus particles per cell of which $\sim 25 \%$ are infectious) [72].

The intracellular location of virus protein glycosylation is unknown, although gold-labeled Vp54 antibody only reacts with proteins in the virus assembly centers (Graves, M.V., Gurnon, J.R. and Van Etten, J.L., unpublished data). This finding suggests that Vp54 might acquire its glycans in the virus assembly centers. 


\section{How are sugars added to Vp54?}

The structures of the glycans attached to Vp54 are unknown and we are happy to collaborate with anyone who wants to solve their structures. The prediction is that the glycan structures differ from typical glycans because it is unlikely that the host ER and Golgi apparatus are involved in adding sugars to PBCV- 1 Vp54. Vp54 lacks a recognizable ER signal peptide and so presumably, it does not enter the ER. However, one can ask the question: are the sugars added to Vp54 either sequentially or are they synthesized independently of Vp54, possibly on a lipid carrier, and then attached to the protein in a single step? A slight variation of these two possibilities is to synthesize a core glycan(s) independently of the protein and attach it to Vp54. This core glycan(s), which could be either larger or smaller than the final product, is processed further after attachment to the protein.

To distinguish between these possibilities $\left[2,6-{ }^{3} \mathrm{H}\right]$-galactose was added to cells at the time of PBCV-1 infection and cells collected at various times p.i. [We are aware that galactose can be converted to other products.] After disrupting the cells, SDS-soluble materials were separated on SDS-PAGE, and labeled material viewed via fluorography (Fig. 3) (Graves, M.V. and Van Etten, J.L., unpublished data). Vp54 first appears between 60 and 90 min p.i. and is a tight homogeneous band suggesting that the fully synthesized glycans are transferred to the Vp54 peptide "en mass" [Note: the Vp54 equivalent from antigenic variant EPA-1 is easily distinguished from wild-type Vp54 even though it is only 2230 Da smaller; the slightly smaller variant Vp54 from mutant E11 can be distinguished as well (Fig. 3)] It is interesting that a slowly migrating radioactive smear is present at the top of the gel first appearing between 15 and 30 min p.i., prior to the appearance of Vp54; the smear increases in intensity to $90 \mathrm{~min}$ p.i. and then becomes less intense as Vp54 intensifies. Could this "smear" be an intermediate(s) in the synthesis of the Vp54 glycan? Interestingly, the appearance of this smear correlates with the expression of A64R, as well as the other putative glycosyltransferases; each of the glycosyltransferase gene transcripts begin to be detected at 20 to $40 \mathrm{~min}$ p.i. (Table 3; [Yanai-Balser, G.M. et al., manuscript in preparation]). Note: the smear is absent in normal cells even after 5 $\mathrm{h}$ of continuous labeling (Fig. 3, lane U). 


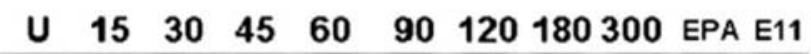

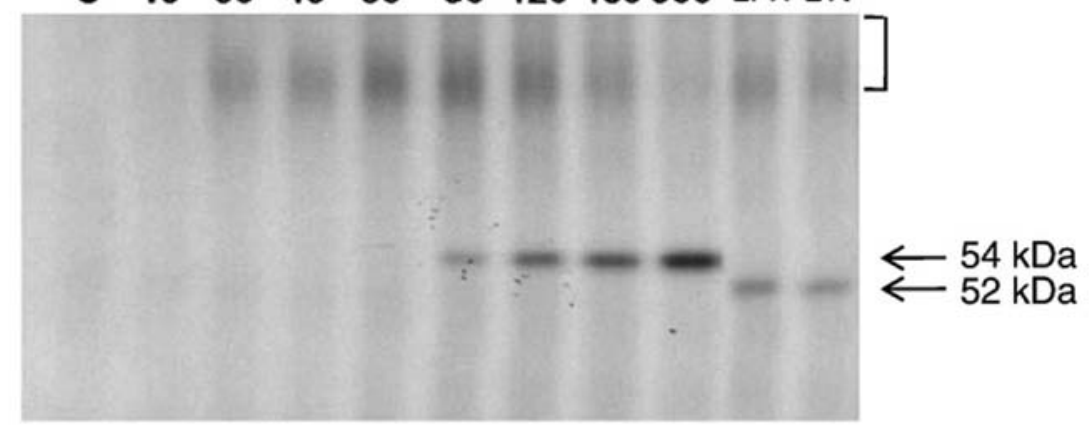

Fig. 3. Total proteins isolated from virus PBCV-1 infected Chlorella NC64A at the indicated times ( $\mathrm{min}$ ) p.i. The infection was done in the presence of [3H]-galactose. Uninfected (lane U) cells were labeled for $300 \mathrm{~min}$. Lanes labeled EPA and E11 contain total proteins from cells at $120 \mathrm{~min}$ after infection with antigenic variant viruses EPA-1 and E11. The slower migrating "smear" described in the text is indicated by the bracket. Proteins were separated on a 7.5\% SDS-PAGE, and labeled proteins visualized by autoradiography (Graves, M.V. and Van Etten, J.L., unpublished data).

Assuming the Vp54 glycan precursors are transferred "en mass", one predicts that they are attached to a lipid carrier such as either undecaprenol-phosphate, which is the carrier for bacterial peptidoglycans and cell surface polysaccharides [77], or dolicholdiphosphate, which is the carrier in eukaryotic cells [67]. It is unlikely that dolicholphosphate is the carrier because tunicamycin, which interferes with the transfer of UDP-N-acetylglucoseamine to dolicholphosphate [64], has no effect on either PBCV-1 replication or Vp54 synthesis, even at concentrations three times that required to inhibit host chlorella growth [55].

\section{Glycosyltransferases coded by other chlorella viruses}

Polyconal antibodies to wild-type PBCV-1 and its antigenic variants do not react with many other independent virus isolates that infect Chlorella NC64A (NC64A viruses). This lack of crossreactivity suggests that the major capsid proteins from these nonreacting viruses have different glycans and as such these viruses code unique glycosyltransferases. In addition, two other related virus/ chlorella systems are available for study, viruses that infect Chlorella Pbi (Pbi viruses) and Chlorella SAG 3.83 (SAG viruses). None of the Pbi viruses or SAG viruses reacts with PBCV-1 antiserum. 
Table 4. Glycosyltransferase-like genes encoded by six viruses that infect three Chlorella species.

\begin{tabular}{|c|c|c|c|c|c|c|c|c|c|c|c|}
\hline \multicolumn{2}{|l|}{$P B C V-1$} & \multicolumn{2}{|l|}{$N Y-2 A$} & \multicolumn{2}{|l|}{ AR158 } & \multicolumn{2}{|l|}{ MT325 } & \multicolumn{2}{|l|}{ FR483 } & \multicolumn{2}{|l|}{ ATCV-1 } \\
\hline$C D S$ & $a a$ & $C D S$ & $a a$ & $C D S$ & $a a$ & $C D S$ & $a a$ & $C D S$ & $a a$ & $C D S$ & $a a$ \\
\hline A064R & 632 & - & - & - & - & - & - & - & - & - & - \\
\hline $\mathrm{A} 111 / 114 \mathrm{R}$ & 860 & B159R & 860 & C150R & 860 & M467R & 849 & N472R & 848 & Z120R & 846 \\
\hline A219/222/226R & 677 & - & - & $\mathrm{C} 265 \mathrm{R}$ & 634 & M721L & 604 & N715L & 629 & Z425R & 612 \\
\hline A473L & 517 & - & - & - & - & M186R & 520 & N191R & 520 & Z178L & 533 \\
\hline A546L & 321 & B763L & 405 & C661L & 396 & - & - & - & - & - & - \\
\hline- & - & B618R & 270 & C559R & 270 & - & - & - & - & - & - \\
\hline- & - & - & - & - & - & - & - & - & - & Z417L & 553 \\
\hline- & - & - & - & - & - & - & - & - & - & Z667L & 546 \\
\hline - & - & - & - & - & - & - & - & - & - & Z823R & 553 \\
\hline
\end{tabular}

CDS's listed in the source line across are considered to be orthologs. The amino acid sequences of each of them are available at http://GreenGene.uml.edu. Viruses PBCV-1, NY-2A and AR158 infect Chlorella NC64A, viruses MT325 and FR483 infect Chlorella Pbi and virus ATCV-1 infects Chlorella SAG 3.83.

We recently sequenced and annotated five additional chlorella viruses, two more NC64A viruses [78], two Pbi viruses [79], and one SAG virus [48]. These newly sequenced viruses encode from 3 to 6 putative glycosyltransferases (Table 4). The PBCV-1 glycosyltransferase $\mathrm{A} 111 / 114 \mathrm{R}$ is the only protein with an ortholog in all of the viruses. The orthologs B618R and C559R from NC64A viruses NY-2A and AR158, respectively, produce a high Blast score with $\mathrm{N}$-acetyglucosaminyltransferases, suggesting that $\mathrm{N}$-acetylglucosamine might be present in their major capsid proteins. The major capsid proteins from NY-2A and AR158 clearly differ from PBCV-1 Vp54 because neither virus reacts with antibodies to PBCV-1 or any of its antigenic variants. The sugar components of the major capsid proteins have not been determined for any of these 5 newly sequenced viruses. The prediction is that the sugars will probably differ both qualitatively and quantitatively from PBCV-1.

\section{Implications}

The chlorella viruses contain genes encoding a variety of glycosyltransferases, most of which, if not all, lack a recognizable ER signal peptide. Furthermore, hundreds of these viruses can be isolated easily from natural sources. We assume that different glycosyltransferases use different 
sugars and form distinct linkages. Because all of the virus-encoded glycosyltransferases lack ER signaling domains and most of them lack transmembrane domains, the virus-encoded recombinant glycosyltransferases are likely to be soluble, which makes them easier to purify and use as reagents for adding sugars to proteins.

\section{Conclusion}

Collectively, the results indicate that glycosylation of the PBCV-1 major capsid protein differs from that of other viruses and that glycosylation probably occurs independently of the ER and Golgi apparatus. Furthermore, most, if not all, of the machinery to carry out this glycosylation is encoded by the virus. These conclusions lead to many questions including:

i) could Vp54 glycosylation reflect an ancestral pathway that existed prior to ER and Golgi formation in eukaryotic cells?

ii) Is PBCV-1 glycosylation similar to that of 0 -antigen-containing bacteria, which add a precise number of sugars to the basal core polysaccharide [80].

iii) Are the Vp54 glycan precursors synthesized and assembled on a lipid carrier?

iv) How much diversity in the number of glycosyltransferases and glycan structures exists among chlorella viruses?

v) What is the ultimate function of the capsid protein glycan?

Acknowledgments - Many people have worked on glycosylation of the PBCV-1 major capsid protein, often without recognition. In addition, to the co-authors on our publications on the subject, we would like to acknowledge Dwight Burbank, Tae-Jin Choi, Mike Gretz, Eric Koh, Michela Tonetti, and Gianluca Damonte for their contributions. Work in the Van Etten laboratory was partially supported by NIH grant GM32441 as well as grant P20-RR15635 from the Centers of Biomedical Research Excellence Program of the National Center for Research Resources. 


\section{References}

[1] R.W. Doms, R.A. Lamb, J.K. Rose, A. Helenius, Folding and assembly of viral membrane proteins, Virology 193 (1993) 545-562.

[2] D.M. Knipe, Virus-host cell interactions, 3rd ed, in: B.N. Fields, D.M. Knipe, P.M. Howley, R.M. Chanock, J.L. Melnick, T.P. Monath, B. Roizman, S.E. Straus (Eds.), Fields Virology, Lippincott-Raven Publ., Philadelphia, 1996, pp. 273-299.

[3] S. Olofsson, J.E.S. Hansen, Host cell glycosylation of viral glycoproteins: a battlefield for host defense and viral resistance, Scand. J. Infect Dis. 30 (1998) 435-440.

[4] D.J. Vigerust, V.L. Shepherd, Virus glycosylation: role in virulence and immune interactions, Trends Microbiol. 15 (2007) 211-218.

[5] N. Markine-Goriaynoff, L. Gillet, J.L. Van Etten, H. Korres, N. Verma, A. Vanderplasschen, Glycosyltransferases encoded by viruses, J. Gen. Virol. 85 (2004) 2741-2754.

[6] H. Gram, W. Ruger, The $\alpha$-glucosyltransferases of bacteriophages T2, T4, and T6. A comparison of their primary structures, Mol. Gen. Genet. 202 (1986) 467-470.

[7] P.S. Freemont, W. Ruger, Crystallization and preliminary X-ray studies of T4 phage $\beta$-glucosyltransferase, J. Mol. Biol. 203 (1988) 525-626.

[8] M. Winkler, W. Ruger, Cloning and sequencing of the genes of $\beta$-glucosyl-HMC- $\alpha$ glucosyl-transferases of bacteriophages T2 and T6, Nucleic Acids Res. 21 (1993) 1500.

[9] D.R. O’Reilly, Baculovirus-encoded ecdysteroid UDP-glucosyltransferases, Insect Biochem. Mol. Biol. 25 (1995) 541-550.

[10] A. Vanderplasschen, N. Markine-Goriaynoff, P. Lomonte, et al., A multipotential $\beta$ - 1, 6-N-acetylglucosaminyl-transferase is encoded by bovine herpes virus type 4, Proc. Natl. Acad. Sci. USA 97 (2000) 5756-5761.

[11] N. Markine-Goriaynoff, J.P. Georgin, M. Goltz, et al., The core $2 \beta-1$, 6-Nacetylglucosaminyltransferase- mucin encoded by bovine herpesvirus 4 was acquired from an ancestor of the African buffalo, J. Virol. 77 (2003) 1784-1792.

[12] N. Markine-Goriaynoff, L. Gillet, O.A. Karlsen, et al., The core $2 \beta-1$, 6-Nacetylglucosaminyl- transferase-M encoded by bovine herpesvirus 4 is not essential for virus replication despite contributing to post-translational modifications of structural proteins, J. Gen. Virol. 85 (2004) 355-367.

[13] C.L. Alfonso, E.R. Tulman, Z. Lu, E. Oma, G.F. Kutish, D.L. Rock, The genome of Melanoplus sanguinipes entomopoxvirus, J. Virol. 73 (1999) 533-552.

[14] A.L. Bawden, K.J. Glassberg, J. Diggans, R. Shaw, W. Farmerie, R.W. Moyer, Complete genomic sequence of the Amsacta moorei entomopoxvirus: analysis and comparison with other poxviruses, Virology 274 (2000) 120-139.

[15] N. Delaroque, D.G. Muller, G. Bothe, T. Pohl, R. Knippers, W. Boland, The complete DNA sequence of the Ectocarpus siliculosus virus genome, Virology 287 (2001) 112-132. 
[16] E. Derelle, C. Ferraz, M.L. Escande, S. Eychenie, R. Cooke, G. Piganeau, Y. Desdevises, L. Bellec, H. Moreau, N. Grimsley, Life-cycle and genome of OtV5, a large DNA virus of the pelagic marine unicellular green alga Ostreococcus tauri, PLoS ONE 3 (2008) e2250.

[17] D. Raoult, S. Audic, C. Robert, C. Abergel, P. Renesto, H. Ogata, B. La Scola, M. Suzan, J.M. Claverie, The 1.2 megabase genome sequence of Mimivirus, Science 306 (2004) 1344-1350.

[18] G. Vestergaard, R. Aramayo, T. Basta, M. Haring, X. Peng, K. Brugger, L. Chen, R. Rachel, N. Boisset, R.A. Garrett, D. Prangishvili, Structure of the acidianus filamentous virus 3 and genomics of related archaeal lipothrixviruses, J. Virol. 82 (2008) 371-381.

[19] X. Xiang, L. Chen, X. Huang, Y. Luo, Q. She, L. Huang, Sulfolobus tengchongensis spindle-shaped virus STSV1: interactions and genomic features, J. Virol. 79 (2005) 8677-8686.

[20] J.L. Van Etten, Unusual life style of giant chlorella viruses, Annu. Rev. Genet. 37 (2003) 153-195.

[21] T. Yamada, H. Onimatsu, J.L. Van Etten, Chlorella viruses, in: K. Maramorosch, A.J. Shatkin (Eds.), Advances in Virus Research, vol. 66, Elsevier Inc., 2006, pp. 293-366.

[22] D.D. Dunigan, L.A. Fitzgerald, J.L. Van Etten, Phycodnaviruses: a peek at genetic diversity, Virus Res. 117 (2006) 119-132.

[23] W.H. Wilson, J.L. Van Etten, M.J. Allen, The Phycodnaviridae: the story of how tiny giants rule the world, in: J.L. Van Etten (Ed.), Lesser Known Large dsDNA Viruses, Springer, Berlin, 2008, pp. 1-42.

[24] J.A. Fuhrman, Marine viruses and their biogeochemical and ecological effects, Nature 399 (1999) 541-548.

[25] S.W. Wilhelm, C.A. Suttle, Viruses and nutrient cycles in the sea - viruses play critical roles in the structure and function of aquatic food webs, Bioscience 49 (1999) 781-784.

[26] C.A. Suttle, Marine viruses - major players in the global ecosystem, Nat. Rev. Microbiol. 5 (2005) 801-812.

[27] K.E. Wommack, R.R. Colwell, Viroplankton: viruses in aquatic ecosystems, Microbiol. Mol. Biol. Rev. 64 (2000) 69-114.

[28] L.M. Iyer, L. Aravind, E.V. Koonin, Common origin of four diverse families of large eukaryotic DNA viruses, J. Virol. 75 (2001) 11720-11734.

[29] L.M. Iyer, S. Balaji, E.V. Koonin, L. Aravind, Evolutionary genomics of nucleocytoplasmic large DNA viruses, Virus Res. 117 (2006) 156-184.

[30] D. Raoult, S. Audic, C. Robert, et al., The 1.2-megabase genome sequence of mimivirus, Science 306 (2004) 1344-1350.

[31] L.P. Villarreal, V.R. DeFilippis, A hypothesis for DNA viruses as the origin of eukaryotic replication proteins, J. Virol. 74 (2000) 7079-7084.

[32] L.P. Villarreal, Viruses and the evolution of life, American Society Microbiology Press, Washington, D.C., 2005395 pp. 
[33] Y. Xia, D.E. Burbank, L. Uher, D. Rabussay, J.L. Van Etten, Restriction endonuclease activity induced by PBCV-1 virus infection of a chlorella-like green alga, Mol. Cell. Biol. 6 (1986) 1430-1439.

[34] Y. Xia, D.E. Burbank, L. Uher, D. Rabussay, J.L. Van Etten, IL-3A virus infection of a chlorella-like green alga induces a DNA restriction endonuclease with novel sequence specificity, Nucleic Acids Res. 15 (1987) 6075-6090.

[35] 0.V. Lavrukhin, J.M. Fortune, T.G. Wood, et al., Topoisomerase II from chlorella virus PBCV-1. Characterization of the smallest known type II topoisomerase, J. Biol. Chem. 275 (2000) 6915-6921.

[36] B. Plugge, S. Gazzarrini, M. Nelson, et al., A potassium channel protein encoded by chlorella virus PBCV-1, Science 287 (2000) 1641-1644.

[37] Y. Li, Z. Lu, L. Sun, S. Ropp, G.F. Kutish, D.L. Rock, J.L. Van Etten, Analysis of 74 kb of DNA located at the right end of the 330-kb chlorella virus PBCV-1 genome, Virology 237 (1997) 360-377.

[38] P.L. DeAngelis, W. Jing, M.V. Graves, D.E. Burbank, J.L. Van Etten, Hyaluronan synthase of chlorella virus PBCV-1, Science 278 (1997) 1800-1803.

[39] M.V. Graves, D.E. Burbank, R. Roth, J. Heuser, P.L. DeAngelis, J.L. Van Etten, Hyaluronan synthesis in virus PBCV-1 infected chlorella-like green algae, Virology 257 (1999) 15-23.

[40] P.L. DeAngelis, Hyaluronan synthases: fascinating glycosyltransferases from vertebrates, bacterial pathogens, and algal viruses, Cell. Mol. Life Sci. 56 (1999) 670-682.

[41] P.L. DeAngelis, Evolution of glycosaminoglycans and their glycosyltransferases: Implications for the extracellular matrices of animals and the capsules of pathogenic bacteria, Anat. Rec. 268 (2002) 317-326.

[42] D. Landstein, M.V. Graves, D.E. Burbank, P.L. DeAngelis, J.L. Van Etten, Chlorella virus PBCV-1 encodes functional glutamine:fructose-6-phosphate amidotransferase and UDP-glucose dehydrogenase enzymes, Virology 250 (1998) 388-396.

[43] T. Kawasaki, M. Tanaka, M. Fujie, S. Usami, K. Sakai, T. Yamada, Chitin synthesis in chlorovirus CVK2-infected chlorella cells, Virology 302 (2002) 123-131.

[44] G.W. Gooday, A.M. Humphreys, W.H. McIntosh, Role of chitinases in fungal growth, in: R. Muzzarelli, C. Jeuniaus, G.W. Gooday (Eds.), Chitin in Nature and Technology, Plenum Press, New York, 1986, pp. 83-91.

[45] T. Yamada, T. Kawasaki, Microbial synthesis of hyaluronan and chitin: New approaches, J. Biosci. Bioeng. 99 (2005) 521-528.

[46] M. Tonetti, D. Zanardi, J.R. Gurnon, et al., Chlorella virus PBCV-1 encodes two enzymes involved in the biosynthesis of GDP-L-fucose and GDP-D-rhamnose, J. Biol. Chem. 278 (2003) 21672-21677.

[47] F. Fruscione, L. Sturla, G. Duncan, et al., Differential role of NADP+ and NADPH in the activity and structure of GDP-D-mannose 4, 6-dehydratase from two chlorella viruses, J. Biol. Chem. 283 (2008) 184-193.

[48] L.A. Fitzgerald, M.V. Graves, X. Li, et al., Sequence and annotation of the 288-kb ATCV-1 virus that infects an endosymbiotic chlorella strain of the heliozoon Acanthocystis turfacea, Virology 362 (2007) 350-361. 
[49] L. Sun, B. Adams, J.R. Gurnon, Y. Ye, J.L. Van Etten, Characterization of two chitinase genes and one chitosanase gene encoded by chlorella virus PBCV-1, Virology 263 (1999) 376-387.

[50] L. Sun, J.R. Gurnon, B.J. Adams, M.V. Graves, J.L. Van Etten, Characterization of a $\beta$ - 1, 3-glucanase encoded by chlorella virus PBCV-1, Virology 276 (2000) $27-36$.

[51] I. Sugimoto, H. Onimatsu, M. Fujie, S. Usami, T. Yamada, vAL-1, a novel polysaccharide lyase encoded by chlorovirus CVK2, FEBS Lett. 559 (2004) 51-56.

[52] M. Furuta, J.O. Schrader, H.S. Schrader, et al., Chlorella virus PBCV-1 encodes a homolog of the bacteriophage T4 UV damage repair gene, DenV, Appl. Environ. Microbiol. 63 (1997) 1551-1556.

[53] A.K. McCullough, M.T. Romberg, S. Nyaga, et al., Characterization of a novel cissyn and trans-syn II pyrimidine dimer glycosylase/AP lyase from a eukaryotic algal virus, PBCV-1, J. Biol. Chem. 273 (1998) 13136-13142.

[54] M.V. Graves, R.H. Meints, Characterization of the major capsid protein and cloning of its gene from algal virus PBCV-1, Virology 188 (1992) 198-207.

[55] Q. Que, Y. Li, I.N. Wang, L.C. Lane, W.G. Chaney, J.L. Van Etten, Protein glycosylation and myristylation in chlorella virus PBCV-1 and its antigenic variants, Virology 203 (1994) 320-327.

[56] X. Yan, N.H. Olson, J.L. Van Etten, M. Bergoin, M.G. Rossmann, T.S. Baker, Structure and assembly of large lipid-containing dsDNA viruses, Nat. Struct. Biol. 7 (2000) 101-103.

[57] A.A. Simpson, N. Nandhagopal, J.L. Van Etten, M.G. Rossmann, Structural analyses of Phycodnaviridae and Iridoviridae requires extension of the rules governing quasi-symmetry, Acta Crystallogr. D59 (2003) 2053-2059.

[58] M.V. Cherrier, V.A. Kostyuchenko, C. Xiao, V.D. Bowman, A.J. Battisti, X. Yan, P.R. Chipman, T.S. Baker, J.L. Van Etten, M.G. Rossmann, An icosahedral algal virus has a complex unique vertex decorated by a spike, Proc. Natl. Acad. Sci. USA 106 (2009) 11085-11089.

[59] I.N. Wang, Y. Li, Q. Que, M. Bhattacharya, L.C. Lane, W.G. Chaney, J.L. Van Etten, Evidence for virus-encoded glycosylation specificity, Proc. Natl. Acad. Sci. USA 90 (1993) 3840-3844.

[60] M.V. Graves, C.T. Bernadt, R. Cerny, J.L. Van Etten, Molecular and genetic evidence for a virus-encoded glycosyltransferase involved in protein glycosylation, Virology 285 (2001) 332-345.

[61] J.L. Van Etten, D.E. Burbank, D. Kuczmarski, R.H. Meints, Virus infection of culturable chlorella-like algae and development of a plaque assay, Science 219 (1983) 994-996.

[62] N. Nandhagopal, A. Simpson, J.R. Gurnon, et al., The structure and evolution of the major capsid protein of a large, lipid-containing, DNA virus, Proc. Natl. Acad. Sci. USA 99 (2002) 14758-14763.

[63] F. Maley, R.B. Trimble, A.L. Tarentino, T.H. Plummer, Characterization of glycoproteins and their associated oligosaccharides through the use of endoglycosidases, Anal. Biochem. 180 (1989) 195-204. 
[64] A.D. Elbein, Inhibitors of the biosynthesis and processing of N-linked oligosaccharide chains, Ann. Rev. Biochem. 56 (1987) 497-534.

[65] F. Wieland, R. Heitzer, W. Schaefer, Asparaginylglucose: novel type of carbohydrate linkage, Proc. Natl. Acad. Sci. USA 80 (1983) 5470-5474.

[66] R. Schreiner, E. Schnabel, F. Wieland, Novel glycosylation in eukaryotes: laminin contains the linkage unit beta-glucosylasparagine, J. Cell Biol. 124 (1994) 1071-1081.

[67] G. Reuter, H.J. Gabius, Eukaryotic glycosylation: whim of nature or multipurpose tool, Cell. Mol. Life Sci. 55 (1999) 368-422.

[68] K. Bruckner, L. Perez, H. Clausen, S. Cohen, Glycosyltransferase activity of Fringe modulates Notch-Delta interactions, Nature 406 (2000) 411-415.

[69] Y.P. Yan, J. Schultz, M. Mlodzik, P. Bork, Secreted fringe-like signaling molecules may be glycosyltransferases, Cell 88 (1997) 9-11.

[70] Y. Zhang, Y. Xiang, J.L. Van Etten, M.G. Rossmann, Structure and function of a chlorella virus PBCV-1 encoded glycosyltransferase, Structure 15 (2007) 1031-1039.

[71] H. Onimatsu, K. Suganuma, S. Uenoyama, T. Yamada, C-terminal repetitive motifs in Vp130 present at the unique vertex of the chlorovirus capsid are essential for binding to the host chlorella cell wall, Virology 353 (2006) 432-442.

[72] R.H. Meints, K. Lee, D.E. Burbank, J.L. Van Etten, Infection of a chlorella-like alga with the virus, PBCV-1: Ultrastructural studies, Virology 138 (1984) 341-346.

[73] A.M. Schuster, L. Girton, D.E. Burbank, J.L. Van Etten, Infection of a chlorellalike alga with the virus PBCV-1: Transcriptional studies, Virology 148 (1986) 181-189.

[74] T. Kawasaki, M. Tanaka, M. Fujie, S. Usami, T. Yamada, Immediate early genes expressed in chlorovirus infections, Virology 318 (2004) 214-223.

[75] R. Grabherr, P. Strasser, J.L. Van Etten, The DNA polymerase gene from chlorella viruses PBCV-1 and NY-2A contains an intron with nuclear splicing sequences, Virology 188 (1992) 721-731.

[76] J.L. Van Etten, D.E. Burbank, J. Joshi, R.H. Meints, DNA, synthesis in a chlorellalike alga following infection with the virus PBCV-1, Virology 134 (1984) 443-449.

[77] C.R. Raetz, C. Whitfield, Lipopolysaccharide endotoxins, Ann. Rev. Biochem. 71 (2002) 635-700.

[78] L.A. Fitzgerald, M.V. Graves, X. Li, T. Feldblyum, W.C. Nierman, J.L. Van Etten, Sequence and annotation of the 369-kb NY-2A and the 345-kb AR158 viruses that infect Chlorella NC64A, Virology 358 (2007) 472-484.

[79] L.A. Fitzgerald, M.V. Graves, X. Li, T. Feldblyum, J. Hartigan, J.L. Van Etten, Sequence and annotation of the 314-kb MT325 and the 321-kb FR483 viruses that infect Chlorella Pbi, Virology 358 (2007) 459-471.

[80] D.A. Bastin, G. Stevenson, P.K. Brown, A. Haase, P.R. Reeves, Repeat unit polysaccharides of bacteria: a model for polymerization resembling that of ribosomes and fatty acid synthetase, with a novel mechanism for determining chain length, Mol. Microbiol. 7 (1993) 725-734. 